\title{
Near-Infrared Emission CulnS/ZnS Quantum Dots: All-in-One Theranostic Nanomedicines with Intrinsic Fluorescence/ Photoacoustic Imaging for Tumor Phototherapy
}

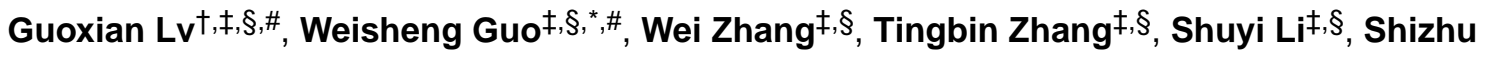 \\ Chen", Ahmed Shaker Eltahan $\ddagger, \S$, Dongliang Wang ${ }^{\ddagger, \S}$, Yuqing Wang ${ }^{\ddagger} \S$, Jinchao Zhang", \\ Paul C. Wang ${ }^{\perp}$, Jin Chang ${ }^{\dagger},{ }^{*}$, and Xing-Jie Liang ${ }^{\ddagger, \S,},{ }^{*}$ \\ †School of Materials Science and Engineering, School of Life Sciences, Tianjin University, Tianjin \\ Engineering Center for Micro-Nano Biomaterials and Detection-Treatment Technology, Tianjin \\ 300072, People's Republic of China \\ ‡Laboratory of Controllable Nanopharmaceuticals, Chinese Academy of Sciences (CAS) Center \\ for Excellence in Nanoscience and CAS Key Laboratory for Biomedical Effects of Nanomaterials \\ and Nanosafety, National Center for Nanoscience and Technology of China, Beijing 100190, \\ People's Republic of China \\ SUniversity of Chinese Academy of Sciences, Beijing 100049, People's Republic of China \\ "College of Chemistry \& Environmental Science, Key Laboratory of Medicinal Chemistry and \\ Molecular Diagnosis of Ministry of Education, Chemical Biology Key Laboratory of Hebei \\ Province, Hebei University, Baoding 071002, People's Republic of China \\ ${ }^{\perp}$ Laboratory of Molecular Imaging, Department of Radiology, Howard University, Washington, \\ D.C. 20060 , United States
}

\begin{abstract}
Many theranostic nanomedicines (NMs) have been fabricated by packaging imaging and therapeutic moieties together. However, concerns about their potential architecture instability and pharmacokinetic complexity remain major obstacles to their clinical translation. Herein, we demonstrated the use of CuInS/ZnS quantum dots (ZCIS QDs) as "all-in-one" theranostic nanomedicines that possess intrinsic imaging and therapeutic capabilities within a well-defined nanostructure. ZCIS QDs were exploited for multispectral optical tomography (MSOT) imaging and synergistic PTT/PDT therapy. Due to the intrinsic fluorescence/MSOT imaging ability of the ZCIS QDs, their size-dependent distribution profiles were successfully visualized at tumor sites in
\end{abstract}

\footnotetext{
"Corresponding Authors. (W. Guo): guows@ @nanoctr.cn., (J. Chang): jinchang @tju.edu.cn. (X.-J. Liang): liangxj@ nanoctr.cn.

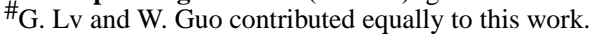

ASSOCIATED CONTENT

Supporting Information

The Supporting Information is available free of charge on the ACS Publications website at DOI: 10.1021/acsnano.6b05419.

Detailed supplementary experimental section, photothermal effect curves, absorbance spectrum of RNO solution without ZCIS NMs, fluorescence stability of ZCIS NMs, hydrodynamic diameter distributions, TEM images of ZCIS NMs, and blood biochemistry analysis figures (PDF)

The authors declare no competing financial interest.
} 
vivo. Our results showed that the smaller nanomedicines (ZCIS NMs-25) have longer tumor retention times, higher tumor uptake, and deeper tumor penetration than the larger nanomedicines (ZCIS NMs-80). The ability of ZCIS QDs to mediate photoinduced tumor ablation was also explored. Our results verified that under a single $660 \mathrm{~nm}$ laser irradiation, the ZCIS NMs had simultaneous inherent photothermal and photodynamic effects, resulting in high therapy efficacy against tumors. In summary, the ZCIS QDs as "all-in-one" versatile nanomedicines allow high therapeutic efficacy as well as noninvasively monitoring tumor site localization profiles by imaging techniques and thus hold great potential as precision theranostic nanomedicines.

\section{Graphical Abstract}

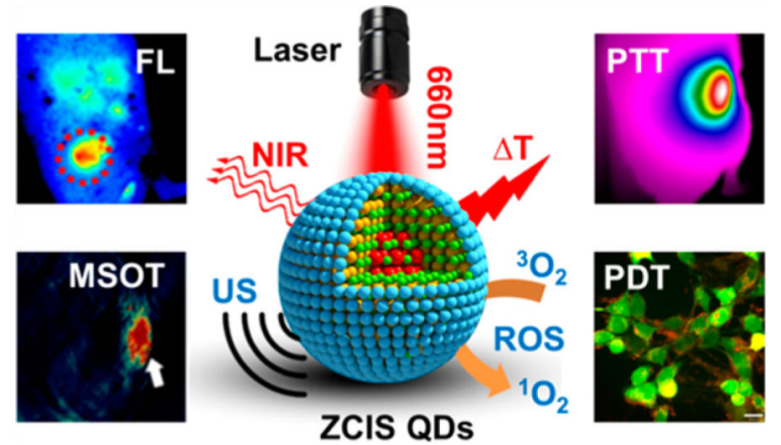

\section{Keywords}

CuInS/ZnS quantum dots; photoacoustic imaging; multispectral optical tomography; photothermal therapy; photodynamic therapy

Parallel advances in nanotechnology, imaging, chemistry, and biology in recent years have resulted in the emergence of theranostic nanomedicines as promising agents to deliver personalized medicine for disease- and patient-specific diagnosis and treatment. ${ }^{1-4}$ By integrating therapeutic and imaging components into a single nanoformulation, theranostic nanomedicines allow precise disease diagnosis, assessment of treatment efficacy, and realtime evaluation of pharmacokinetics by imaging techniques. ${ }^{5,6}$ Despite these conceptual advances, theranostic nanomedicines are still at an early stage of development and have not yet achieved clinical application because of concerns mainly regarding their safety and nanostructure/surface-dependent pharmacokinetics. ${ }^{7-10}$ Notably, theranostic nanomedicines are usually fabricated by encapsulating or conjugating both therapeutic and imaging moieties together, which gives rise to concerns about unstable nanostructures, altered surfaces, and increased pharmacokinetic complexity. ${ }^{2,5}$ Therefore, it is essential to fabricate "all-in-one" theranostic nanomedicines with intrinsic imaging and therapeutic capabilities, ${ }^{1}$ allowing high therapeutic efficacy as well as noninvasive monitoring of the localization profile of the nanomedicine at the tumor site by imaging techniques.

Among the various biomedical imaging modalities, fluorescence imaging is the most widely employed in (pre)clinical research due to its low cost and short acquisition time. Despite its poor spatial resolution and limited penetration depth for in vivo imaging, fluorescence imaging is of particular interest for single-molecule tracking and imaging of cellular 
constituents thanks to its outstanding sensitivity. ${ }^{11,12}$ On the other hand, multispectral optoacoustic tomography (MSOT) is a hybrid imaging modality that overcomes the optical diffusion limitation by integrating the spectral selectivity of molecular excitation with the high resolution of ultrasound detection based on the photoacoustic (PA) effect. ${ }^{13-15}$ MSOT imaging thus holds great promise for the visualization of physiological and pathological processes at the molecular level with deep tissue penetration and fine spatial resolution. Therefore, it would be advantageous to integrate fluorescence and MSOT imaging into a single nanomedicine, where highly sensitive fluorescence imaging enables detection of the behavior of the nanomedicine in vitro, while MSOT imaging allows noninvasive visualization of the nanomedicine accumulation profile at the tumor site in vivo. ${ }^{15}$ However, the nanoparticles (NPs) used for MSOT imaging, such as carbon NPs, ${ }^{16,17} \mathrm{CuS} \mathrm{NPs},{ }^{18-20}$ gold nanorods, ${ }^{21,22}$ and nanoporphyrin, ${ }^{23}$ usually show strong light excitation absorption and would thereby result in serious fluorescence quenching when integrated with fluorophores. ${ }^{24}$ Therefore, it is still a major challenge to achieve theranostic nanomedicines with intrinsic fluorescence/MSOT dual-modal imaging ability.

Activatable theranostics have been receiving enormous attention as an emerging clinical approach to early diagnosis, monitoring, and prognostic evaluation of diseases via imaging techniques followed by tailored therapy. ${ }^{25,26}$ In particular, photomediated therapeutic approaches, such as photodynamic therapy (PDT) and photothermal therapy (PTT), $14,23-25,27-31$ have been increasingly recognized as promising alternatives to traditional cancer therapies, due to the advantages of spatiotemporal selectivity, reduced side effects, and minimal invasiveness. PTT, which involves photothermal conversion agents that induce hyperthermia by exposure to a laser, can result in ablation of cancer cells depending on the increase in the local temperature in the tumor. Typically, an elevated temperature $\left(>70{ }^{\circ} \mathrm{C}\right)$ is needed for efficient hyperthermal destruction of a tumor, ${ }^{32}$ which might cause undesirable destruction of nanomedicines and serious collateral damage to normal tissue. On the other hand, PDT employs a photo-sensitizer (PS) that can convert normal oxygen $\left({ }^{3} \mathrm{O}_{2}\right)$ to the reactive and cytotoxic singlet oxygen $\left({ }^{1} \mathrm{O}_{2}\right)$ upon exposure to specific light wavelengths. However, typical PS agents absorb light in the ultraviolet/visible region and suffer from easy photodecomposition. ${ }^{33,34}$ Therefore, it would be advantageous to build theranostic nanomedicines that enable simultaneous PTT and PDT following a single NIR laser exposure, which will achieve high therapeutic efficacy even at a moderate local temperature $\left(<45^{\circ} \mathrm{C}\right)$.

Quantum dots (QDs) have been intensively investigated as theranostic nanomedicines due to their distinct advantages of bright fluorescence, broad excitation spectrum, robust structure, and resistance to photobleaching. ${ }^{35,36}$ However, QDs have seldom been exploited for MSOT imaging and PTT since the first pioneering exploration by Zharov's group. ${ }^{37}$ In this work, we report on highly versatile "all-in-one" theranostic nanomedicines based on CuInS/ZnS (ZCIS) QDs, which have a broad absorption band in the visible region $(600-700 \mathrm{~nm})$. Significantly, the ZCIS QDs exhibited strong NIR emission centered at $730 \mathrm{~nm}$ as well as good MSOT imaging ability and synergistic PTT/PDT effects upon $660 \mathrm{~nm}$ laser irradiation. These properties have rarely been reported to date. Thus, the ZCIS QDs are intrinsically capable of various imaging and therapeutic functions and have successfully been evaluated 
as "all-in-one" theranostic nanomedicines that can be used for fluorescence/MSOT dualmodal imaging and PTT/PDT-mediated tumor ablation (Figure 1A).

\section{RESULTS AND DISCUSSION}

\section{Characterization, Fluorescence, and MSOT Properties of ZCIS NMs}

Our proposed "all-in-one" theranostic nanomedicines (NMs) were made from ZCIS QDs with a surface coating of lipid (DSPE-PEG-2K) and are denoted as ZCIS NMs. The employed ZCIS QDs were synthesized according to our previously reported methods with advantages of low toxicity and NIR emission over conventional CdSe-class QDs. ${ }^{38,39}$ The imaging capabilities of the ZCIS NMs were investigated in detail. As indicated in Figure 1B, the ZCIS NMs were well-dispersed in aqueous medium and showed strong NIR fluorescence emission with a peak at $730 \mathrm{~nm}$. The fluorescence quantum yields were calculated to be $12.2 \%$ using a fluorospectrophotometer equipped with an integrating sphere. Transmission electron microscopy (TEM) observation (Figure 1D) confirmed the uniform size distribution and spherical morphology of the obtained ZCIS QDs and revealed a wellresolved crystal lattice, which indicates robust nanostructure. Notably, a significant absorption band was observed in the NIR region (650-750 nm) (Figure 1C), suggesting potential photoacoustic (PA) imaging ability. Encouraged by this result, we audaciously explored the potential of the ZCIS NMs for PA imaging, despite the fact that similar studies have seldom been carried out on QDs. As presented in Figure 1E and G, ZCIS NMs at various mass concentrations were embedded in agar gel cylinders to create in vitro PA imaging phantoms, which were studied in a MSOT imaging system. The results in Figure 1E reveal that the PA signal was a function of the excitation light wavelength and the ZCIS NM concentration. The ZCIS NMs showed a continuous PA spectrum, with a more intense signal in the shorter wavelength NIR region. This corresponds to the observed optical absorption spectrum in Figure 1C. Quantitative analysis showed that the PA intensity was linearly correlated with the ZCIS NMs concentration from 0 to $200 \mu \mathrm{g} / \mathrm{mL}$, as presented in Figure 1E and F. These findings demonstrated the potential of these versatile ZCIS NMs as theranostic nanomedicines with intrinsic fluorescence/MSOT dual-modal imaging ability.

\section{Photothermal and Photodynamic Effect of ZCIS NMs}

Given the efficient photon absorption of the ZCIS NMs in the NIR region $(650-750 \mathrm{~nm})$, we next wanted to investigated the potential of the nanodots for use in PTT and PDT ablation of tumors. The temperature elevation of aqueous dispersions of the ZCIS NMs at various concentrations $(0-200 \mu \mathrm{g} / \mathrm{mL})$ was monitored under continuous laser irradiation (660 nm, 1 $\mathrm{W} / \mathrm{cm}^{2}$ ) with an infrared thermal camera, as shown in Figure 2A-C. No obvious temperature rise was observed in the control sample of pure water, while the aqueous ZCIS NMs exhibited a clear photothermal effect. Temperature increases of $10-22{ }^{\circ} \mathrm{C}$ were detected after laser irradiation for $10 \mathrm{~min}$, owing to the efficient NIR absorption of the ZCIS NMs. The temperature rise at the end of the $10 \mathrm{~min}$ laser irradiation period was plotted against the ZCIS NM concentration (Figure 2B). The temperature elevation profile flattened out with increasing concentration, which can be attributed to the dependence of the logarithmic absorbance on the fraction of incident radiation. To further study the photothermal stability and transduction efficiency of the ZCIS NMs, an aqueous solution $(200 \mu \mathrm{g} / \mathrm{mL}, 1 \mathrm{~mL})$ was 
continuously illuminated by a $660 \mathrm{~nm}$ laser $\left(1 \mathrm{~W} / \mathrm{cm}^{2}\right)$ until it reached a steady-state temperature, at which point the laser was stopped and the suspension was allowed to cool naturally. The temperature change during the heating-cooling process was monitored for four cycles to derive a heat generation-dissipation curve as shown in Figure 2D. An almost equal temperature elevation of $22{ }^{\circ} \mathrm{C}$ occurred during each laser ON/OFF cycle, suggesting excellent photothermal stability of the ZCIS NMs. On the basis of the photothermal curve in Figure 2D and the quantification method first developed by $\operatorname{Roper}^{40}$ (see method details and Figure S1 in the Supporting Information), the photothermal conversion efficiency was calculated as $11.6 \%$, which is fairly acceptable and comparable to that of Au nanoshells $(13 \%){ }^{41}$

QDs possess several distinct characteristics that make them potentially suitable as photosensitizers. ${ }^{42,43}$ We therefore investigated whether the ZCIS NMs were able to generate reactive oxygen species (ROS) in vitro upon exposure to the same laser treatment $\left(1 \mathrm{~W} / \mathrm{cm}^{2}\right)$. ROS were detected by incubating ZCIS NMs with $p$-nitrosodimethylaniline (RNO), which is subjected to photobleaching and exhibits reduced absorbance at $440 \mathrm{~nm}$ in the presence of ROS. As shown in Figure 2E and Figure S2 (in the Supporting Information), the maximum absorption of the suspension gradually decreased as the laser irradiation time increased, suggesting that ROS was efficiently generated. Notably, equal decreases in absorbance and negligible fluorescence changes (see Figure S3 in the Supporting Information) were observed for each time interval during the 25 min laser irradiation, implying good photo-chemical stability of the ZCIS NMs. These interesting findings demonstrated that the ZCIS NMs are promising nanomedicines that allow simultaneous PTT and PDT upon a single laser exposure.

\section{In Vitro Phototherapy Evaluation and Multicellular Spheroid Penetration}

Encouraged by the promising phototherapy efficiency, we next evaluated the cytotoxicity and in vitro phototherapy efficacy of the ZCIS NMs in the mouse mammary carcinoma cell line $4 \mathrm{~T} 1$. We tested the viability of $4 \mathrm{~T} 1$ cells after exposure to various concentrations of ZCIS NMs for $24 \mathrm{~h}$ with or without laser irradiation. As shown in Figure 3A, even with 200 $\mu \mathrm{g} / \mathrm{mL}$ of ZCIS NMs, the cell viability was almost $90 \%$, which suggested that ZCIS NMs had very low cytotoxicity. To verify the enhanced phototherapy efficacy induced by ZCIS NMs, live and dead cells were differentiated by costaining with calcein AM (green fluorescence; living cells) and PI (red fluorescence; dead cells) after laser treatment (Figure 3C). In the untreated and control (ZCIS NMs only or laser only) groups, all cells displayed green fluorescence, illustrating that ZCIS NMs alone or NIR laser irradiation alone had little effect on the viability of the cells. In contrast, almost all the cells that were incubated with ZCIS NMs and then exposed to NIR laser $\left(1 \mathrm{~W} / \mathrm{cm}^{2}, 10 \mathrm{~min}\right)$ were killed and displayed red fluorescence. As shown in Figure 3B, the phototherapeutic efficacy of the ZCIS NMs was dependent on the irradiation time, with over $95 \%$ of the cells killed after 10 min exposure. On the basis of the above study, we predict that the synergistic PTT and PDT effect of the ZCIS NMs will enhance their efficacy. Next, we examined the intracellular ROS production by ZCIS NMs under NIR laser irradiation using $2^{\prime}, 7^{\prime}$-dichlorofluorescin diacetate (DCFHDA). DCFH-DA is deacetylated to a nonfluorescent compound once it has diffused into cells and is oxidized by ROS to $2^{\prime}, 7^{\prime}$-dichlorofluorescin (DCF), which has fluorescent emission 
at $525 \mathrm{~nm}$. As shown in Figure 3D and Figure S4 (see Supporting Information), confocal laser scanning microscopy (CLSM) observation revealed very weak fluorescence of ROS, when the cells were cultured with phosphate-buffered saline (PBS) or ZCIS NMs only or treated with NIR irradiation alone. In contrast, intense intracellular green fluorescence was observed in cells cultured with ZCIS NMs followed by $660 \mathrm{~nm}$ laser irradiation $\left(1 \mathrm{~W} / \mathrm{cm}^{2}\right.$, $10 \mathrm{~min}$ ), indicating efficient intracellular ROS generation. Meanwhile, the inherent fluorescence of ZCIS NMs allowed us to visualize their intracellular distribution. The NIRemissive ZCIS NMs clearly accumulated in the cytoplasm, leaving the nucleus as a clear zone (Figure S5, see Supporting Information). Together with the results of the cell viability tests, these lines of evidence indicate that the ZCIS NMs are efficient theranostic nanomedicines with intrinsic and synergistic PTT and PDT properties.

\section{In Vivo Fluorescence/MSOT Imaging Guided Tumor-Site Accumulation of ZCIS NMs}

On the basis of the favorable in vitro results, we next used in vivo fluorescence/MSOT dualmodal imaging to evaluate the penetration and retention of ZCIS NMs at the tumor site. Prior to animal experiments, the initially hydrophobic ZCIS QDs were encapsulated into lipid-PEG (DSPE-PEG) micelles, resulting in ZCIS NMs. By varying the amount of DSPEPEG, ZCIS NMs with hydrodynamic diameters (HDs) of 25.3 and $82 \mathrm{~nm}$ were prepared and designated as ZCIS NMs-25 and ZCIS NMs-80, respectively (Figures S6 and S7 in the Supporting Information). As shown in Figure S8 (in the Supporting Information), the temporal evolution of the HD profile was recorded up to 3 days, depicting a good colloidal stability of the ZCIS NMs-25 in water and PBS. These ZCIS NMs were employed to study the effect of size on the retention and penetration profiles of the particles in nude mice bearing $4 \mathrm{~T} 1$ tumors. When the tumor volume reached $100 \mathrm{~mm}^{3}$, the mice were administered with an intravenous (i.v.) injection of ZCIS NMs $(2 \mathrm{mg} / \mathrm{mL}, 200 \mu \mathrm{L})$ and then subjected to NIR fluorescence and MSOT imaging. As shown in Figure 4, ZCIS NMs fluorescence was distributed throughout the body soon after i.v. injection. Between 6 and $48 \mathrm{~h}$ postinjection, the ZCIS NMs gradually accumulated in the tumor site through the enhanced permeability and retention (EPR) effect, resulting in strong signals in the tumor region. The accumulation of ZCIS NMs in tumors was semiquantified in vivo by fluorescence intensity (Figure 4C). The average fluorescence intensity derived from the tumor site increased continuously until $24 \mathrm{~h}$ postinjection, after which it decreased slightly. At $48 \mathrm{~h}$ postinjection, the major organs of the treated mice were harvested for ex vivo fluorescence imaging and tissue biodistribution quantification by ICP-MS. As presented in Figure 4B, distinct fluorescent signals were clearly observed in the tumors despite the strong signals in the liver and spleen. These results were in agreement with the ICP-MS data shown in Figure 4D. Notably, a higher tumor site signal was observed in the mice injected with ZCIS NMs-25 than with ZCIS NMs-80. The quantification analysis shown in Figure 4C and D also indicates that the ZCIS NMs-25 have a longer retention time ( $48 \mathrm{~h}$ ) and higher relative uptake efficiency (5.2 $\pm 1.2 \%)$ than ZCIS NMs-80 ( $24 \mathrm{~h}, 3.2 \pm 0.7 \%)$. This implies that the behavior of the ZCISNMs at the tumor site is size-dependent.

Because fluorescence imaging has relatively poor resolution, we exploited the intrinsic MSOT imaging feature of the ZCIS QDs to carry out a more thorough study of the sizedependent tumor site distribution behaviors of the ZCIS NMs in mice. In vivo MSOT 
imaging is noninvasive and facilitates the acquisition of both anatomical and functional information with deeper penetration and higher spatial resolution. As shown in Figure 4E, the contrast at the tumor site was markedly enhanced from $4 \mathrm{~h}$ postinjection, indicating the efficient passive targeting and MSOT imaging ability of ZCIS NMs in vivo. The PA signal analysis in Figure 4G shows that the PA signals initially rose and then slightly decreased after $24 \mathrm{~h}$ postinjection, a pattern that is similar to the fluorescence profile in Figure 4C. We also focused in more detail on the different information from the tumor area of the two experimental groups (ZCIS NMs-25 and ZCIS NMs-80). As shown in Figure 4E,G, at $24 \mathrm{~h}$ postinjection of ZCIS NMs-25, the PA signal intensity in the tumor was enhanced by over $300 \%$, while the enhancement was less than 200\% in the case of ZCIS NMs-80. This is in accordance with the uptake analysis by ICP-MS in Figure 4C. In the mice injected with ZCIS NMs-25, the tumor was more markedly visible even up to $48 \mathrm{~h}$ postinjection compared to the mice injected with ZCIS NMs-80, suggesting a longer tumor retention time. The tumor penetration activities of ZCIS NMs-25 and ZCIS NMs- 80 are clearly demonstrated by the enlarged orthogonal view of the tumor in Figure 4E. Following ZCIS NMs-80 treatment, the periphery of the tumor showed significant PA signals, but the signals from the interior area of the tumor were weak. In the case of ZCIS NMs-25, intense PA signals were clearly detected throughout the tumor region, demonstrating the deeper penetration and uniform distribution of ZCIS NMs-25 in the tumor. In order to eliminate the possibility that the results were due to tumor necrosis, the mice were subjected to magnetic resonance imaging (MRI), which provides tissue pathological and anatomical information (Figure 4F). The tumors exhibited homogeneous contrast imaging, indicating no necrosis or inflammation. The size-dependent penetration capability of ZCIS NMs was also carefully validated using in vitro multicellular spheroids (MCSs). MCSs are versatile 3D tumor models that are similar to solid tumors based on their morphology and biological properties. ${ }^{44,45}$ As shown in Figure 3E, the CLSM Z-stack scanning results revealed that the red fluorescence of ZCIS NMs-80 was mostly detected on the periphery of the MCSs, while the fluorescence of ZCIS NMs-25 was clearly observed inside the MCSs even at a depth of $110 \mu \mathrm{m}$. This suggests that the smaller ZCIS NMs have a more favorable penetration profile. Taken together, the evidence suggests that ZCIS NMs-25 have an advantageous tumor distribution profile, including longer retention time, higher tumor uptake efficiency, and deeper tumor penetration. This is consistent with previous reports that nanoparticles with sizes ranging from 20 to $50 \mathrm{~nm}$ have favorable accumulation and retention in tumors. ${ }^{14,46}$

\section{In Vivo Phototherapy Efficacy and Toxicity Assessment of ZCIS NMs}

Having precisely monitored the tumor site distribution profiles of the ZCIS NMs, we next assessed their phototherapy efficacy against tumors in vivo. When the tumor volumes reached about $100 \mathrm{~mm}^{3}$, nude mice bearing 4T1 tumors were divided into four treatment groups: PBS injection only; ZCIS NMs-25 injection only; PBS injection and laser irradiation; and ZCIS NMs-25 injection and laser irradiation. The treated mice were i.v. injected with ZCIS NMs at a dose of $40 \mathrm{mg} / \mathrm{kg}$ and then exposed to a $660 \mathrm{~nm}$ laser (1 W/ $\mathrm{cm}^{2}$ ) for $12 \mathrm{~min}$ at $12 \mathrm{~h}$ postinjection. During the laser exposure, thermal images were continuously acquired with an infrared thermal camera to monitor changes in the temperature at the tumor site (Figure 5A,B). Tumors in the mice injected with PBS or ZCIS NMs-25 only showed no temperature change. The temperature of the tumor area in the mice 
treated with ZCIS NMs-25 and laser irradiation rapidly increased to $46{ }^{\circ} \mathrm{C}$ within $6 \mathrm{~min}$. In contrast, the tumor temperature of the mice treated with PBS and laser irradiation showed an increase of $\sim 5^{\circ} \mathrm{C}$. The tumor volumes and body weights of each group of mice were monitored for 12 days (Figure 5C,D). While the tumors in the control groups all increased in size, the tumors in the group treated with ZCIS NMs and laser irradiation showed marked growth inhibition and regression after 12 days, leaving scars at the initial sites. On the basis of our in vitro investigations, we attributed the efficient phototherapy efficacy of the ZCIS NMs to the intrinsic and synergistic PTT and PDT effect of the CIS/ZnS QDs. The tumors from each group were harvested at the end of the experimental period (Figure 5E) and stained with hematoxylin and eosin (H\&E) (Figure 5F). The histochemical analysis suggested obvious necrosis of the QDs+Laser-treated tumor tissues, including features such as nuclear condensation, severe cell shrinkage and loss of contact, and breakdown of the tumor extracellular matrix. On the other hand, no obvious damage to the tumor tissue was observed in the three control groups. Taken together, these results demonstrate that ZCIS NMs are efficient nanomedicines with intrinsic phototherapy effects.

It is essential to assess the safety of any nanomaterial that is intended for use in biomedical applications. Therefore, we further evaluated the in vivo toxicity of ZCIS NMs by H\&E staining of the major organs and blood biochemistry analysis (Figure 5G and Figure S9 in the Supporting Information). No significant difference was observed in the concentrations of hepatic function markers (ALT and AST) and renal function markers (CREA, UA, and BUN) after the ZCIS NMs injections. This suggests that the ZCIS NMs induced negligible renal and hepatic dysfunction. Meanwhile, the H\&E-stained slides did not show any obvious tissue inflammation, cell necrosis, or apoptosis in the heart, liver, spleen, lung, and kidney (Figure 5G), indicating an absence of evident toxic side effects. Collectively, our results verified that ZCIS NMs had low toxicity and could therefore serve as theranostic nanomedicines with dual-imaging and phototherapeutic properties.

\section{CONCLUSION}

In summary, ZCIS NMs QDs were demonstrated as "all-in-one" theranostic nanomedicines with inherent fluorescence/MSOT imaging properties as well as PTT/PDT effects. The ZCIS NMs can simultaneously act as a "spy satellite" and a "precision weapon" for precise diagnosis and efficient destruction of tumors. Due to their efficient absorption in the range of 650-750 nm, ZCIS QDs were successfully used for MSOT imaging and synergistic PTT/PDT therapy. The intrinsic fluorescence/MSOT imaging ability of the ZCIS QDs enabled the noninvasive visualization of their size-dependent distribution profiles at the tumor site in vivo. Our results showed that the smaller nanomedicines (ZCIS NMs-25) have longer tumor retention times, higher tumor uptake, and deeper tumor penetration than the larger nanomedicines (ZCIS NMs-80). In addition to the imaging properties, the ability of ZCIS QDs to mediate photoinduced tumor ablation was intensively explored. Our results verified that the ZCIS NMs delivered simultaneous photothermal and photodynamic effects upon a single $660 \mathrm{~nm}$ laser irradiation, resulting in efficient synergistic phototherapy efficacy against tumors. These nanoparticles still require in-depth research into their pharmacokinetic properties and potential toxicity. Nevertheless, the ZCIS QDs, as versatile 
"all-in-one" nanocrystals, hold great potential as a paradigm for precision theranostic nanomedicines.

\section{EXPERIMENTAL SECTION}

\section{Reagents}

Copper(I) iodide (CuI, 99.9\%), indium(III) acetate ( $\left.\mathrm{In}(\mathrm{OA})_{3}, 99.9 \%\right)$, zinc stearate $(\mathrm{ZnO}$, 12.5-14\%), 1-dodecanethiol (DDT, 98\%), oleylamine (97\%), oleic acid (OA, 90\%), 1octadecene (ODE, 90\%), calcein-AM, propidium iodide (PI), p-nitrosodimethylaniline, and $2^{\prime}, 7^{\prime}$-dichlorodihydrofluorescein diacetate (DCFH-DA) were purchased from SigmaAldrich and used as received without further purification. DSPE-PEG-2K was purchased from Yarebio (Shanghai, China). Deionized water $\left(18.2 \mathrm{M} \Omega \cdot \mathrm{cm}\right.$ resistivity at $\left.25^{\circ} \mathrm{C}\right)$ was used for all tests.

\section{Synthesis of ZCIS QDs}

ZCIS QDs were prepared via a thermal decomposition strategy reported in our previous work. ${ }^{39}$ Typically, $\mathrm{CuI}(0.2 \mathrm{mmol})$, zinc stearate $\left(\mathrm{Zn}(\mathrm{SA})_{2}\right)(0.1 \mathrm{mmol}), \operatorname{In}(\mathrm{Ac})_{3}(0.2 \mathrm{mmol})$, and $\mathrm{OA}(1.0 \mathrm{mmol})$ were mixed with $8.0 \mathrm{~mL}$ of ODE and $1.0 \mathrm{~mL}$ of DDT in a four-neck flask under magnetic stirring. The solution was heated to $100^{\circ} \mathrm{C}$, degassed for $30 \mathrm{~min}$, and subsequently further heated to $240{ }^{\circ} \mathrm{C}$ under an argon atmosphere. The reaction solution was cooled to $150{ }^{\circ} \mathrm{C}$ after $60 \mathrm{~min}$, followed by sequential injections of $2 \mathrm{~mL}$ of sulfur precursor (0.1 $\mathrm{M}$ in ODE/oleylamine, $\mathrm{v} / \mathrm{v} 10: 1)$ and $3 \mathrm{~mL}$ of zinc stearate solution ( $0.1 \mathrm{M}$ in ODE) in three batches with a time interval of $15 \mathrm{~min}$. Then the temperature was increased to $220{ }^{\circ} \mathrm{C}$ for $60 \mathrm{~min}$. The as-prepared ZCIS QDs were added with ethanol until the solution became turbid and then collected by centrifugation $(8000 \mathrm{rpm}, 20 \mathrm{~min}$ ). Two more purifications with hexane/ethanol (1:3) cosolvents by centrifugation were performed. The obtained ZCIS QDs were finally dispersed into chloroform.

\section{Preparation of ZCIS NMs}

The initially prepared ZCIS QDs were hydrophobic and biologically modified with $\mathrm{mPEG}_{2000}$-DSPE via an ultrasonication emulsion strategy prior to biomedical applications. For the typical preparation of ZCIS NMs-25, $4 \mathrm{~mL}$ of water containing $8 \mathrm{mg}$ of $\mathrm{mPEG}_{2000^{-}}$ DSPE was placed under an ultrasonic transducer. Then $1 \mathrm{~mL}$ of ZCIS QDs $(2 \mathrm{mg})$ solution in chloroform was added dropwise into the aqueous solution under ultrasonication $(100 \mathrm{~W})$. After ultrasonicating for $5 \mathrm{~min}$, the obtained QDs/lipid emulsion was evaporated to remove chloroform followed by sonication for 5 min, resulting in a clear aqueous ZCIS NMs-25 solution. The resulting solution was filtered to remove large aggregations and centrifuged three times to remove excess $\mathrm{mPEG}_{2000}$-DSPE. The final solution was kept at $4{ }^{\circ} \mathrm{C}$ for further use. The mass concentration was determined by lyophilization. ZCIS NMs-80 can be prepared at the same conditions except increasing $\mathrm{mPEG}_{2000}$-DSPE to $16 \mathrm{mg}$.

\section{Penetration of ZCIS NMs-25 and ZCIS NMs-80 in MCSs}

MCSs were transferred to ultralow attachment 24-well plates (Corning, USA) and incubated in RPMI 1640 medium containing ZCIS NMs $(100 \mu \mathrm{g} / \mathrm{mL})$ for $12 \mathrm{~h}$. Then, the MCSs were 
collected and washed with PBS three times and observed by a Zeiss LSM 710 inverted laser confocal scanning microscope.

\section{In Vivo Fluorescence/MSOT Imaging}

ZCIS NMs ( $200 \mu \mathrm{L}, 2 \mathrm{mg} / \mathrm{mL}$ ) were i.v. injected into the $4 \mathrm{~T} 1$ tumor bearing BALB/c nude mice. The fluorescence images were acquired on a CRI Maestro system using the red excitation channel and an NIR filter at different postinjection time points. The images were unmixed by the Maestro software. All mice were euthanized after the $48 \mathrm{~h}$ imaging. All major organs including tumors were collected and subjected to ex vivo imaging.

In vivo photoacoustic imaging was acquired on the multispectral optical tomography system (MSOT inVision 128, iThera medical, Germany). Photoacoustic signals were detected under different excitation wavelengths $(680-900 \mathrm{~nm})$. The two excitation wavelengths were used to measure oxygenated and deoxygenated hemoglobin at 850 and $750 \mathrm{~nm}$, respectively. MSOT signals before injection were recorded as a control.

\section{In Vivo Phototherapy Assessment}

The 4T1 tumor-bearing BALB/c nude mice (female, 6 weeks old) were i.v. injected with ZCIS NMs- 25 at a dose of $40 \mathrm{mg} / \mathrm{kg}$ when the tumor diameter reached $\sim 6 \mathrm{~mm}$. An equal volume of PBS buffer solution was injected into the mice as a control. After $12 \mathrm{~h}$ postinjection, the tumor sites of both experimental and control groups were exposed to a 660 $\mathrm{nm}$ laser $\left(1 \mathrm{~W} / \mathrm{cm}^{2}\right.$ for $\left.12 \mathrm{~min}\right)$. During the irradiation process, the temperature of the mice was continuously monitored by a Fluke (Ti400) thermal imaging camera.

\section{Supplementary Material}

Refer to Web version on PubMed Central for supplementary material.

\section{Acknowledgments}

The authors gratefully acknowledge the financial support from the Chinese National Natural Science Foundation key project (31430031, 81601603, 51373117, and 51573128) and National Distinguished Young Scholars grant (31225009). The authors also appreciate the support by the external cooperation program of BIC, Chinese Academy of Science, Grant No. 121D11KYSB20130006, and the "Strategic Priority Research Program" of the Chinese Academy of Sciences Grant No. XDA09030301.

\section{REFERENCES}

1. Ma XW, Zhao YL, Liang XJ. Theranostic Nanoparticles Engineered for Clinic and Pharmaceutics. Acc. Chem. Res. 2011; 44:1114-1122. [PubMed: 21732606]

2. Lammers T, Aime S, Hennink WE, Storm G, Kiessling F. Theranostic Nanomedicine. Acc. Chem. Res. 2011; 44:1029-1038. [PubMed: 21545096]

3. Xie J, Lee S, Chen XY. Nanoparticle-Based Theranostic Agents. Adv. Drug Delivery Rev. 2010; 62:1064-1079.

4. Jokerst JV, Gambhir SS. Molecular Imaging with Theranostic Nanoparticles. Acc. Chem. Res. 2011; 44:1050-1060. [PubMed: 21919457]

5. Janib SM, Moses AS, MacKay JA. Imaging and Drug Delivery Using Theranostic Nanoparticles. Adv. Drug Delivery Rev. 2010; 62:1052-1063.

6. Chen F, Ehlerding EB, Cai W. Theranostic Nanoparticles. J. Nucl. Med. 2014; 55:1919-1922. [PubMed: 25413134] 
7. Xue X, Xu J, Wang PC, Liang X-J. Subcellular Behaviour Evaluation of Nanopharmaceuticals with Aggregation-Induced Emission Molecules. J. Mater. Chem. C. 2016; 4:2719-2730.

8. Singh TG, Dhiman S, Rehni AK. Formulation and Evaluation of Nanopharmaceuticals in Drug Delivery. Future Med. 2013; 11:80-95.

9. Schütz CA, Juillerat-Jeanneret L, Mueller H, Lynch I, Riediker M. Therapeutic Nanoparticles in Clinics and Under Clinical Evaluation. Nanomedicine. 2013; 8:449-467. [PubMed: 23477336]

10. Ma X, Gong N, Zhong L, Sun J, Liang X-J. Future of Nanotherapeutics: Targeting the Cellular Sub-Organelles. Biomaterials. 2016; 97:10-21. [PubMed: 27155363]

11. Tanenbaum ME, Gilbert LA, Qi LS, Weissman JS, Vale RD. A Protein-Tagging System for Signal Amplification in Gene Expression and Fluorescence Imaging. Cell. 2014; 159:635-646. [PubMed: 25307933]

12. Doksani Y, Wu JY, de Lange T, Zhuang X. Super-Resolution Fluorescence Imaging of Telomeres Reveals TRF2-dependent T-loop Formation. Cell. 2013; 155:345-356. [PubMed: 24120135]

13. Wang LV, Hu S. Photoacoustic Tomography: In Vivo Imaging from Organelles to Organs. Science. 2012; 335:1458-1462. [PubMed: 22442475]

14. Pu K, Shuhendler AJ, Jokerst JV, Mei J, Gambhir SS, Bao Z, Rao J. Semiconducting Polymer Nanoparticles as Photoacoustic Molecular Imaging Probes in Living Mice. Nat. Nanotechnol. 2014; 9:233-239. [PubMed: 24463363]

15. Li K, Liu B. Polymer-Encapsulated Organic Nanoparticles for Fluorescence and Photoacoustic Imaging. Chem. Soc. Rev. 2014; 43:6570-6597. [PubMed: 24792930]

16. Yang K, Feng L, Shi X, Liu Z. Nano-Graphene in Biomedicine: Theranostic Applications. Chem. Soc. Rev. 2013; 42:530-547. [PubMed: 23059655]

17. Ge J, Jia Q, Liu W, Guo L, Liu Q, Lan M, Zhang H, Meng X, Wang P. Red-Emissive Carbon Dots for Fluorescent, Photoacoustic, and Thermal Theranostics in Living Mice. Adv. Mater. 2015; 27:4169-4177. [PubMed: 26045099]

18. Zhou M, Li J, Liang S, Sood AK, Liang D, Li C. CuS Nanodots with Ultrahigh Efficient Renal Clearance for Positron Emission Tomography Imaging and Image-Guided Photothermal Therapy. ACS Nano. 2015; 9:7085-7096. [PubMed: 26098195]

19. Wang S, Riedinger A, Li H, Fu C, Liu H, Li L, Liu T, Tan L, Barthel MJ, Pugliese G. Plasmonic Copper Sulfide Nanocrystals Exhibiting Near-Infrared Photothermal and Photodynamic Therapeutic Effects. ACS Nano. 2015; 9:1788-1800. [PubMed: 25603353]

20. Chen F, Hong H, Goel S, Graves SA, Orbay H, Ehlerding EB, Shi S, Theuer CP, Nickles RJ, Cai W. In Vivo Tumor Vasculature Targeting of CuS@MSN Based Theranostic Nanomedicine. ACS Nano. 2015; 9:3926-3934. [PubMed: 25843647]

21. Song J, Yang X, Jacobson O, Huang P, Sun X, Lin L, Yan X, Niu G, Ma Q, Chen X. Ultrasmall Gold Nanorod Vesicles with Enhanced Tumor Accumulation and Fast Excretion from the Body for Cancer Therapy. Adv. Mater. 2015; 27:4910-4917. [PubMed: 26198622]

22. Song J, Yang X, Jacobson O, Lin L, Huang P, Niu G, Ma Q, Chen X. Sequential Drug Release and Enhanced Photothermal and Photoacoustic Effect of Hybrid Reduced Graphene Oxide-Loaded Ultrasmall Gold Nanorod Vesicles for Cancer Therapy. ACS Nano. 2015; 9:9199-9209. [PubMed: 26308265]

23. Li Y, Lin T-y, Luo Y, Liu Q, Xiao W, Guo W, Lac D, Zhang H, Feng C, Wachsmann-Hogiu S. A Smart and Versatile Theranostic Nanomedicine Platform Based on Nanoporphyrin. Nat. Commun. 2014; 5:4712-4726. [PubMed: 25158161]

24. Huang P, Rong P, Jin A, Yan X, Zhang MG, Lin J, Hu H, Wang Z, Yue X, Li W. Dye-Loaded Ferritin Nanocages for Multimodal Imaging and Photothermal Therapy. Adv. Mater. 2014; 26:6401-6408. [PubMed: 25123089]

25. Kumar A, Kumar S, Rhim W-K, Kim G-H, Nam J-M. Oxidative Nanopeeling Chemistry-Based Synthesis and Photodynamic and Photothermal Therapeutic Applications of Plasmonic Core-Petal Nanostructures. J. Am. Chem. Soc. 2014; 136:16317-16325. [PubMed: 25386786]

26. Huang P, Gao Y, Lin J, Hu H, Liao HS, Yan XF, Tang YX, Jin A, Song JB, Niu G, Zhang GF, Horkay F, Chen XY. Tumor-Specific Formation of Enzyme-Instructed Supramolecular SelfAssemblies as Cancer Theranostics. ACS Nano. 2015; 9:9517-9527. [PubMed: 26301492] 
27. Liu Y, Liu Y, Bu W, Cheng C, Zuo C, Xiao Q, Sun Y, Ni D, Zhang C, Liu J. Hypoxia Induced by Upconversion Based Photodynamic Therapy: Towards Highly Effective Synergistic Bioreductive Therapy in Tumors. Angew. Chem. 2015; 127:8223-8227.

28. Lin J, Wang M, Hu H, Yang X, Wen B, Wang Z, Jacobson O, Song J, Zhang G, Niu G. Multimodal-Imaging-Guided Cancer Phototherapy by Versatile Biomimetic Theranostics with UV and $\gamma$-Irradiation Protection. Adv. Mater. 2016; 28:3273-3279. [PubMed: 26928972]

29. Zhao P, Zheng M, Luo Z, Gong P, Gao G, Sheng Z, Zheng C, Ma Y, Cai L. NIR-Driven Smart Theranostic Nanomedicine for On-Demand Drug Release and Synergistic Antitumour Therapy. Sci. Rep. 2015; 5:14258-14271. [PubMed: 26400780]

30. Ding X, Liow CH, Zhang M, Huang R, Li C, Shen H, Liu M, Zou Y, Gao N, Zhang Z. Surface Plasmon Resonance Enhanced Light Absorption and Photothermal Therapy in the Second NearInfrared Window. J. Am. Chem. Soc. 2014; 136:15684-15693. [PubMed: 25340966]

31. Wang SJ, Huang P, Nie LM, Xing RJ, Liu DB, Wang Z, Lin J, Chen SH, Niu G, Lu GM, Chen XY. Single Continuous Wave Laser Induced Photodynamic/Plasmonic Photothermal Therapy Using Photosensitizer-Functionalized Gold Nanostars. Adv. Mater. 2013; 25:3055-3061. [PubMed: 23404693]

32. Liu J, Zheng X, Yan L, Zhou L, Tian G, Yin W, Wang L, Liu Y, Hu Z, Gu Z. Bismuth Sulfide Nanorods as a Precision Nanomedicine for In Vivo Multimodal Imaging-Guided Photothermal Therapy of Tumor. ACS Nano. 2015; 9:696-707. [PubMed: 25561009]

33. Zhuang XX, Ma XW, Xue XD, Jiang Q, Song LL, Dai LR, Zhang CQ, Jin SB, Yang KN, Ding BQ, Wang PC, Liang XJ. A Photosensitizer-Loaded DNA Origami Nanosystem for Photodynamic Therapy. ACS Nano. 2016; 10:3486-3495. [PubMed: 26950644]

34. Hu D, Sheng Z, Gao G, Siu F, Liu C, Wan Q, Gong P, Zheng H, Ma Y, Cai L. Activatable Albumin-Photosensitizer Nanoassemblies for Triple-Modal Imaging and Thermal-Modulated Photodynamic Therapy of Cancer. Biomaterials. 2016; 93:10-19. [PubMed: 27061266]

35. Michalet X, Pinaud F, Bentolila L, Tsay J, Doose S, Li J, Sundaresan G, Wu A, Gambhir S, Weiss S. Quantum Dots for Live Cells, In Vivo Imaging, and Diagnostics. Science. 2005; 307:538-544. [PubMed: 15681376]

36. Gao X, Cui Y, Levenson RM, Chung LW, Nie S. In Vivo Cancer Targeting and Imaging with Semiconductor Quantum Dots. Nat. Biotechnol. 2004; 22:969-976. [PubMed: 15258594]

37. Shashkov EV, Everts M, Galanzha EI, Zharov VP. Quantum Dots as Multimodal Photoacoustic and Photothermal Contrast Agents. Nano Lett. 2008; 8:3953-3958. [PubMed: 18834183]

38. Guo W, Sun X, Jacobson O, Yan X, Min K, Srivatsan A, Niu G, Kiesewetter DO, Chang J, Chen X. Intrinsically Radioactive $\left[{ }^{64} \mathrm{Cu}\right] \mathrm{CuInS} / \mathrm{ZnS}$ Quantum Dots for PET and Optical Imaging: Improved Radiochemical Stability and Controllable Cerenkov Luminescence. ACS Nano. 2015; 9:488-495. [PubMed: 25549258]

39. Guo W, Chen N, Tu Y, Dong C, Zhang B, Hu C, Chang J. Synthesis of Zn-Cu-In-S/ZnS Core/Shell Quantum Dots with Inhibited Blue-Shift Photoluminescence and Applications for Tumor Targeted Bioimaging. Theranostics. 2013; 3:99-108. [PubMed: 23422883]

40. Roper DK, Ahn W, Hoepfner M. Microscale Heat Transfer Transduced by Surface Plasmon Resonant Gold Nanoparticles. J. Phys. Chem. C. 2007; 111:3636-3641.

41. Hessel CM, Pattani VP, Rasch M, Panthani MG, Koo B, Tunnell JW, Korgel BA. Copper Selenide Nanocrystals for Photothermal Therapy. Nano Lett. 2011; 11:2560-2566. [PubMed: 21553924]

42. Bakalova R, Ohba H, Zhelev Z, Ishikawa M, Baba Y. Quantum Dots as Photosensitizers? Nat. Biotechnol. 2004; 22:1360-1361. [PubMed: 15529155]

43. Samia AC, Chen X, Burda C. Semiconductor Quantum Dots for Photodynamic Therapy. J. Am. Chem. Soc. 2003; 125:15736-15737. [PubMed: 14677951]

44. Li H-J, Du J-Z, Liu J, Du X-J, Shen S, Zhu Y-H, Wang X, Ye X, Nie S, Wang J. Smart Superstructures with Ultrahigh pH-Sensitivity for Targeting Acidic Tumor Microenvironment: Instantaneous Size Switching and Improved Tumor Penetration. ACS Nano. 2016; 10:6753-6761. [PubMed: 27244096]

45. Li H-J, Du J-Z, Du X-J, Xu C-F, Sun C-Y, Wang H-X, Cao Z-T, Yang X-Z, Zhu Y-H, Nie S. Stimuli-Responsive Clustered Nanoparticles for Improved Tumor Penetration and Therapeutic Efficacy. Proc. Natl. Acad. Sci. U. S. A. 2016; 113:4164-4169. [PubMed: 27035960] 
46. Blanco E, Shen H, Ferrari M. Principles of Nanoparticle Design for Overcoming Biological Barriers to Drug Delivery. Nat. Biotechnol. 2015; 33:941-951. [PubMed: 26348965] 

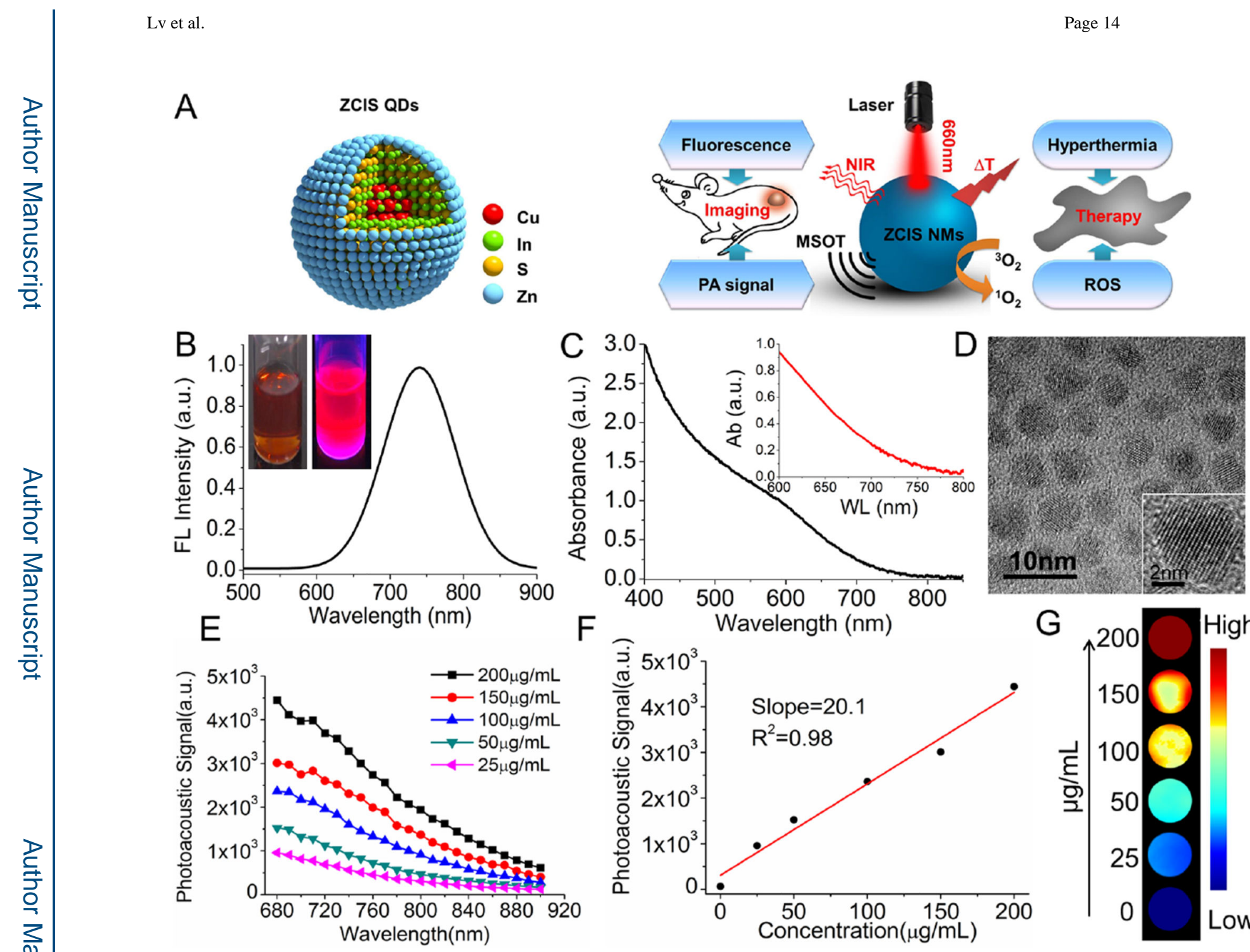

Figure 1.

Scheme and characterization of ZCIS QDs. (A) Illustration of ZCIS QDs used as theranostic nanomedicines with intrinsic fluorescence/MSOT imaging and PTT/PDT therapy abilities. (B) Fluorescence emission spectra (inset: the digital photograph). (C) UV-vis absorption spectrum (inset: enlargement of the absorption from 600 to $800 \mathrm{~nm}$ ). (D) TEM and highresolution TEM (inset) images of ZCIS QDs. (E) Photoacoustic signal spectrum of ZCIS QDs@lipid nanomedicines (designated ZCIS NMs) at various concentrations. (F) Linear relationship between PA signal intensities and concentrations of ZCIS NMs. (G) MSOT imaging phantoms, consisting of various concentrations of ZCIS NMs embedded in agar gel cylinders. The abbreviations in the illustration are as follows: ZCIS QDs (CuInS/ZnS quantum dots), PA signal (photoacoustic signal), NIR (near-infrared), MSOT (multispectral optical tomography), and ROS (reactive oxygen species). 

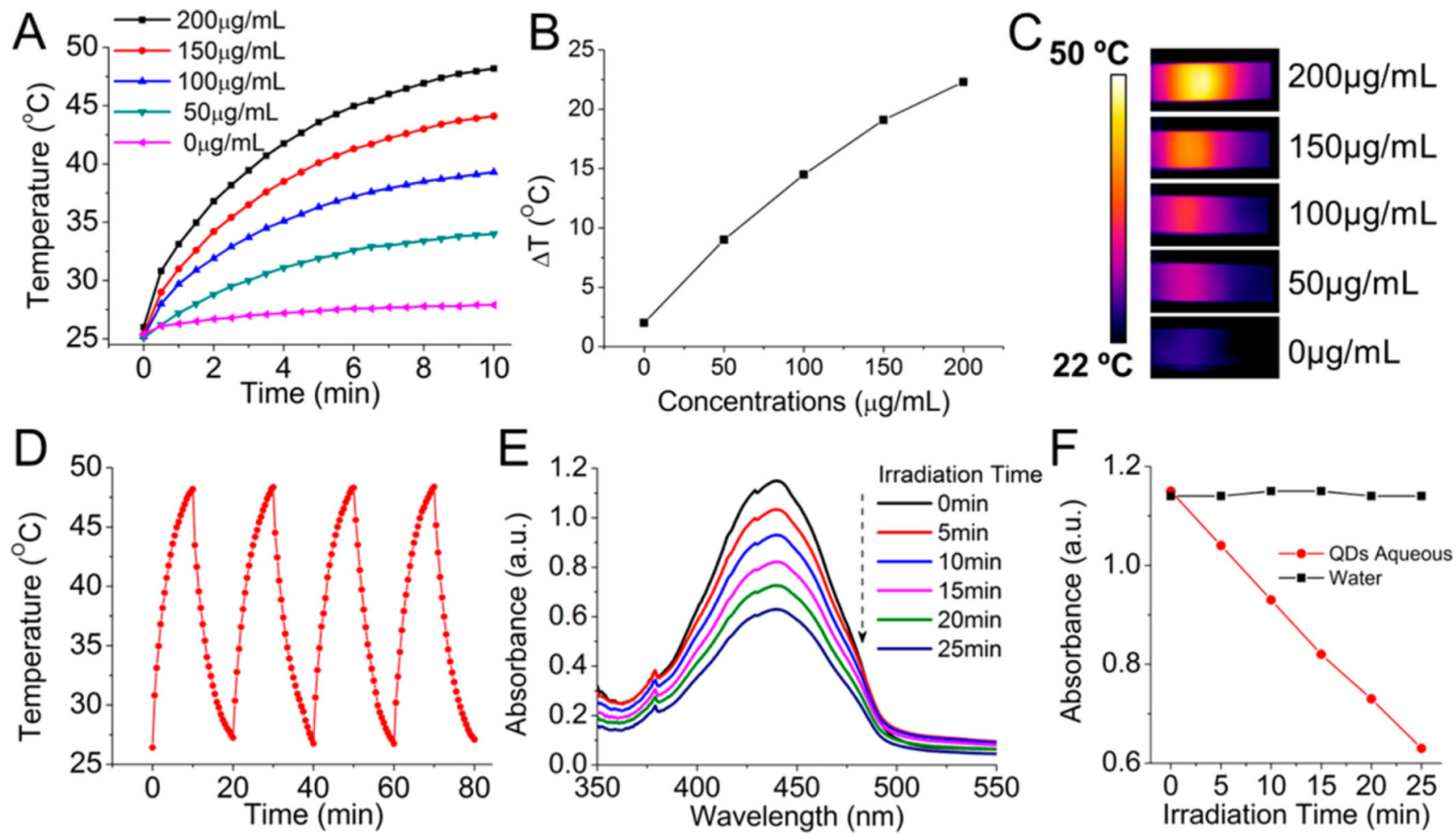

Figure 2.

Photothermal and photodynamic properties of ZCIS NMs. (A) Temperature elevation of the ZCIS NMs in aqueous solution at different concentrations under laser irradiation as a function of irradiation time. (B) Plot of temperature change over a period of 10 min versus the concentration of ZCIS NMs in aqueous solution. (C) Thermal infrared images of ZCIS NMs in aqueous solution in a cuvette under laser irradiation. (D) Temperature elevation of the ZCIS NMs in aqueous solution ( $200 \mu \mathrm{g} / \mathrm{mL}$ ) over four ON/OFF cycles of NIR laser irradiation. (E) Absorbance spectrum of RNO solution containing ZCIS NMs under laser irradiation. (F) Plot showing the change in absorbance of RNO solution at $440 \mathrm{~nm}$ during 25 min of laser exposure (laser irradiation: $660 \mathrm{~nm}, 1 \mathrm{~W} / \mathrm{cm}^{2}$ ). 

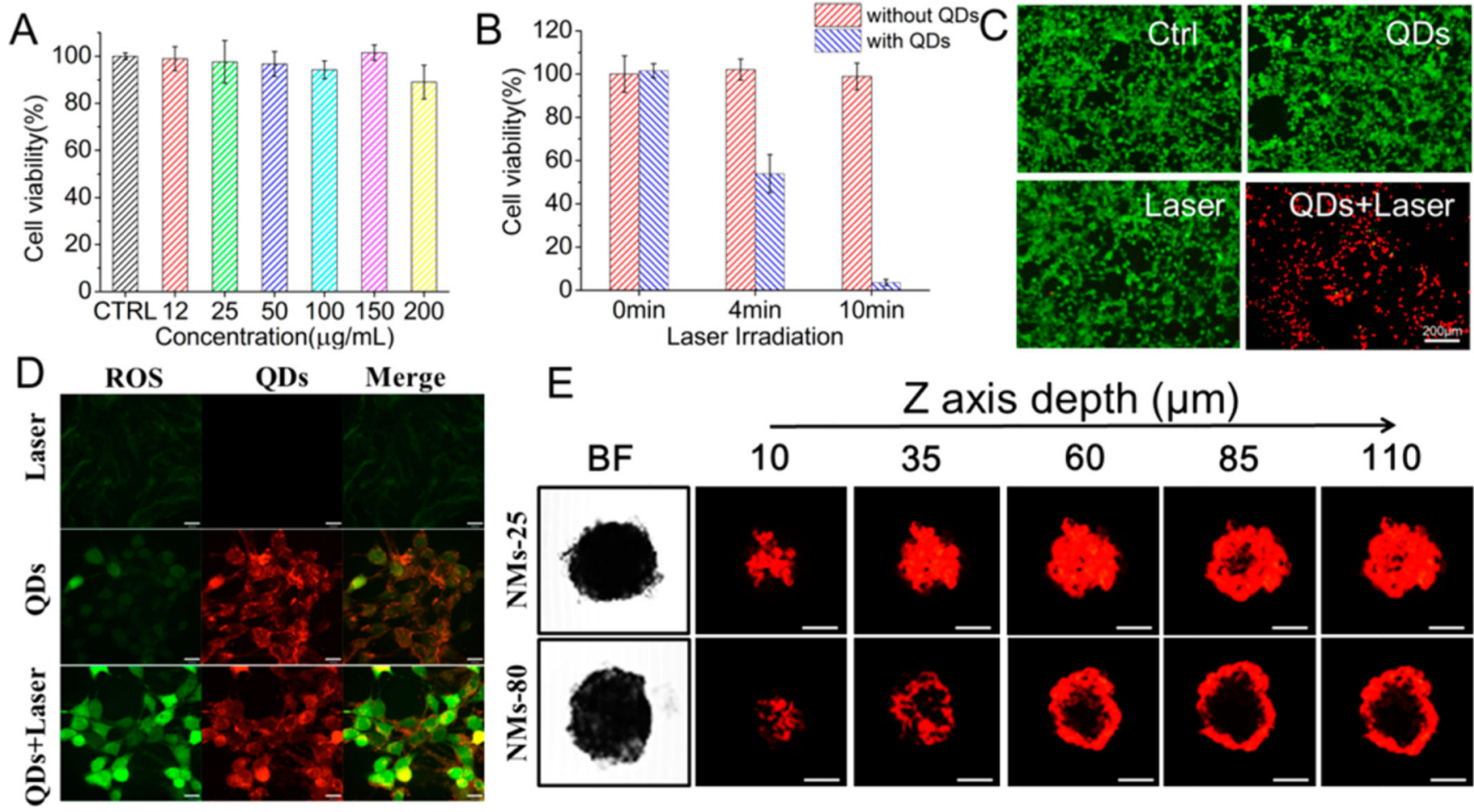

$Z$ axis depth $(\mu \mathrm{m})$

Figure 3.

In vitro cell experiments. (A) Viability of $4 \mathrm{~T} 1$ cells after incubation with ZCIS NMs at various concentrations for $24 \mathrm{~h}$. (B) Viabilities of 4T1 cells after ZCIS NM-induced photoablation under $660 \mathrm{~nm}$ laser irradiation $\left(1 \mathrm{~W} / \mathrm{cm}^{2}\right)$ for different lengths of time. (C) Fluorescence images of calcein AM/PI-costained 4T1 cells with or without ZCIS NM solution $(100 \mu \mathrm{g} / \mathrm{mL})$ after exposure to a $660 \mathrm{~nm}$ laser $\left(1 \mathrm{~W} / \mathrm{cm}^{2}, 10 \mathrm{~min}\right)$. Ctrl: cells with no ZCIS NM/laser treatment; QDs: cells treated with ZCIS NMs alone; Laser: cells treated with laser alone; QDs+Laser: cells treated with both ZCIS NMs and laser irradiation. (D) Confocal fluorescence images of cells treated with laser only (Laser), ZCIS NMs only (QDs), and ZCIS NMs/laser (QDs+Laser). The $660 \mathrm{~nm}$ laser irradiation was at $1 \mathrm{~W} / \mathrm{cm}^{2}$ for $10 \mathrm{~min}$. Cellular ROS generation was detected by DCF fluorescence. Scale bar $=16 \mu \mathrm{m}$. (E) Confocal fluorescence images showing in vitro penetration of ZCIS NMs-25 and ZCIS NMs-80 into 4T1 multicellular spheroids (MCSs). The MCSs were incubated with ZCIS NMs- 25 or ZCIS NMs- 80 for $8 \mathrm{~h}$ and observed by CLSM Z-stack scanning. The surface of the MCSs was defined as $0 \mu \mathrm{m}$. Scale bar $=50 \mu \mathrm{m}$. 


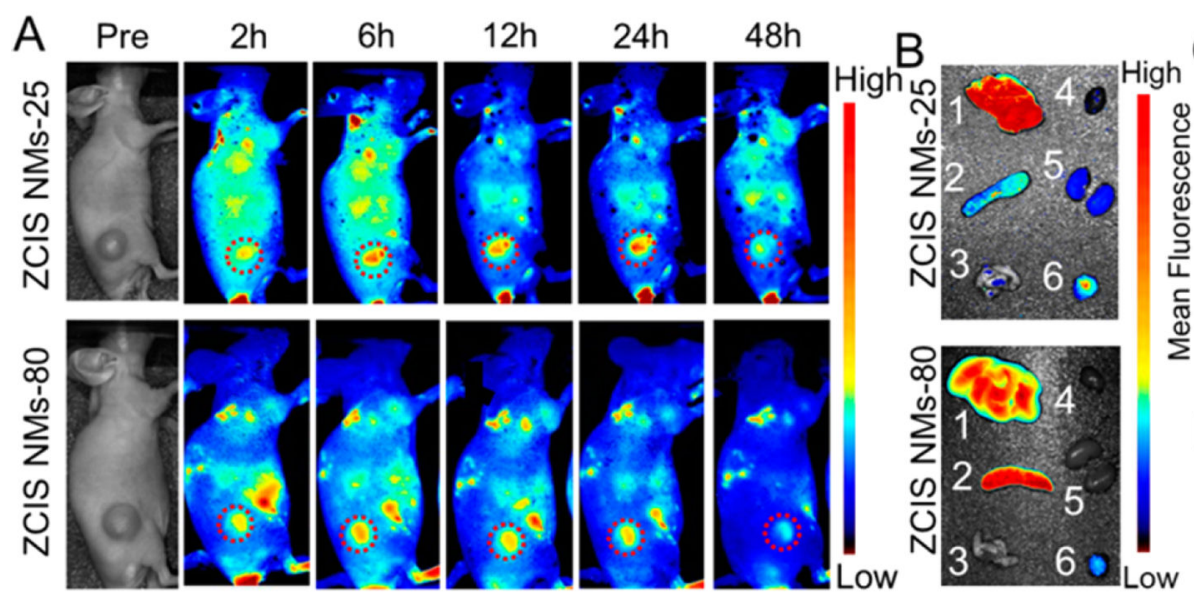

$\mathrm{E}$

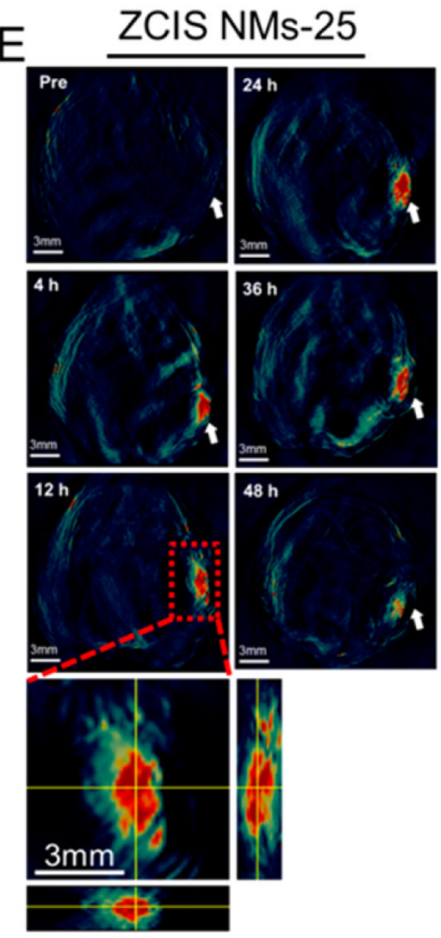

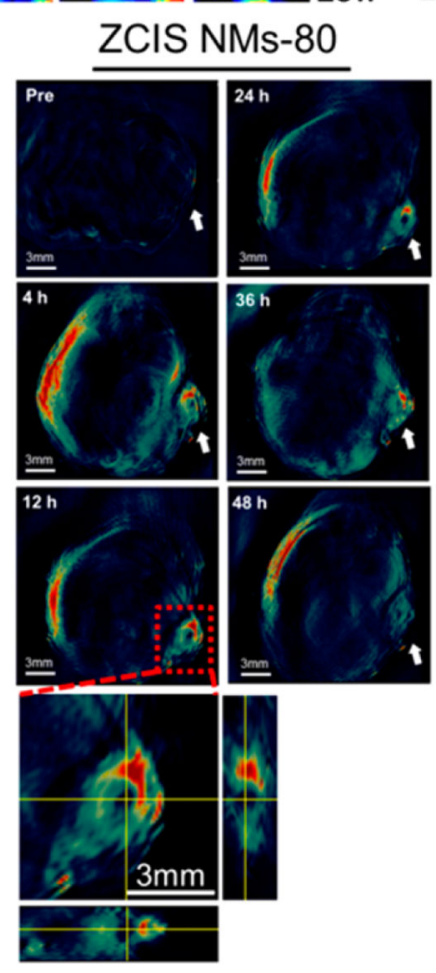

ZCIS NMs-25 ZCIS NMs-80
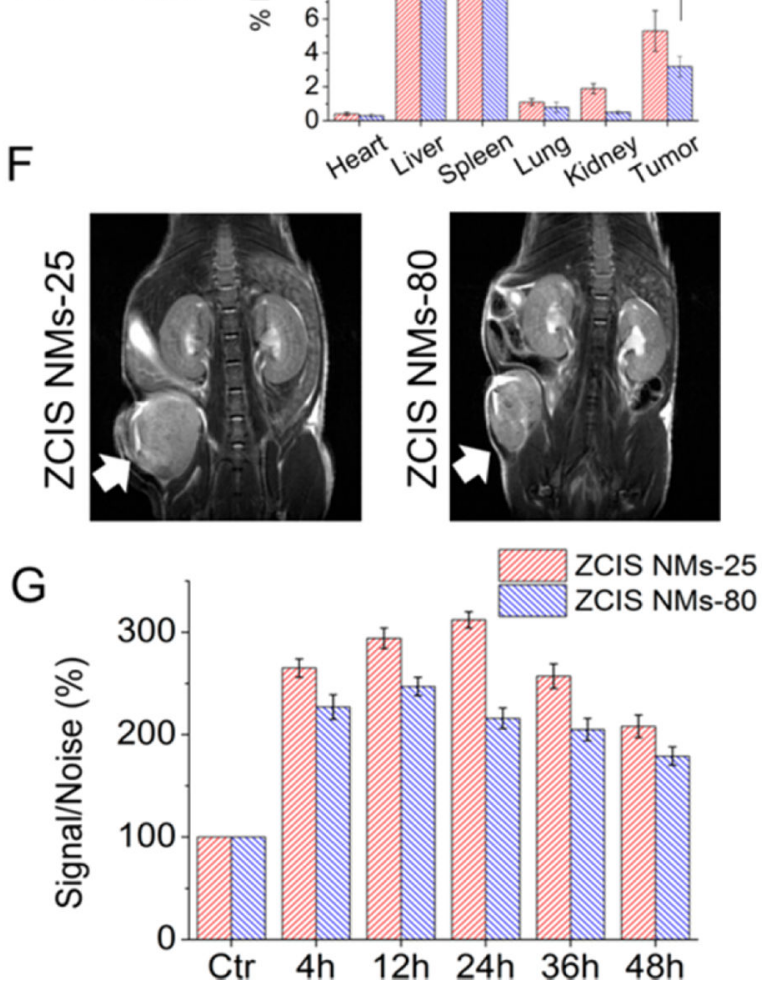

Figure 4.

In vivo fluorescence and MSOT imaging. (A) Real-time in vivo NIR fluorescence images of tumor-bearing mice at various time points after i.v. injections of ZCIS NMs-25 and ZCIS NMs-80. (B) EX vivo NIR fluorescence images of the major organs harvested at $48 \mathrm{~h}$ postinjection (1, liver; 2, spleen; 3, lung; 4, heart; 5, kidney; 6, tumor). (C) Average fluorescence intensities of the tumor areas over $48 \mathrm{~h}$ after injections. (D) Biodistributions of ZCIS NMs-25 and ZCIS NMs-80 in major organs of 4T1 tumor-bearing mice at $48 \mathrm{~h}$ postinjection, determined by ICP-MS analysis. (E) In vivo MSOT images of tumors (arrows) in mice taken at different times after intravenous injection of ZCIS NMs-25 and ZCIS NMs-80. Enlarged orthogonal views of the tumor region are also presented. (F) In vivo MRI images of the tumor-bearing mice acquired at $48 \mathrm{~h}$ postinjection (tumors are indicated by arrows). (G) MSOT signal enhancement in the tumor at different time points postinjection. 
The signal enhancement values were calculated by $\mathrm{SI}_{\text {post }} / \mathrm{SI}_{\text {pre }}$ (SI denotes signal intensity). Each group contained 3 mice. Error bars, mean $\pm \mathrm{SD},{ }^{*} P<0.05$ (two-tailed Student's $t$ test). 

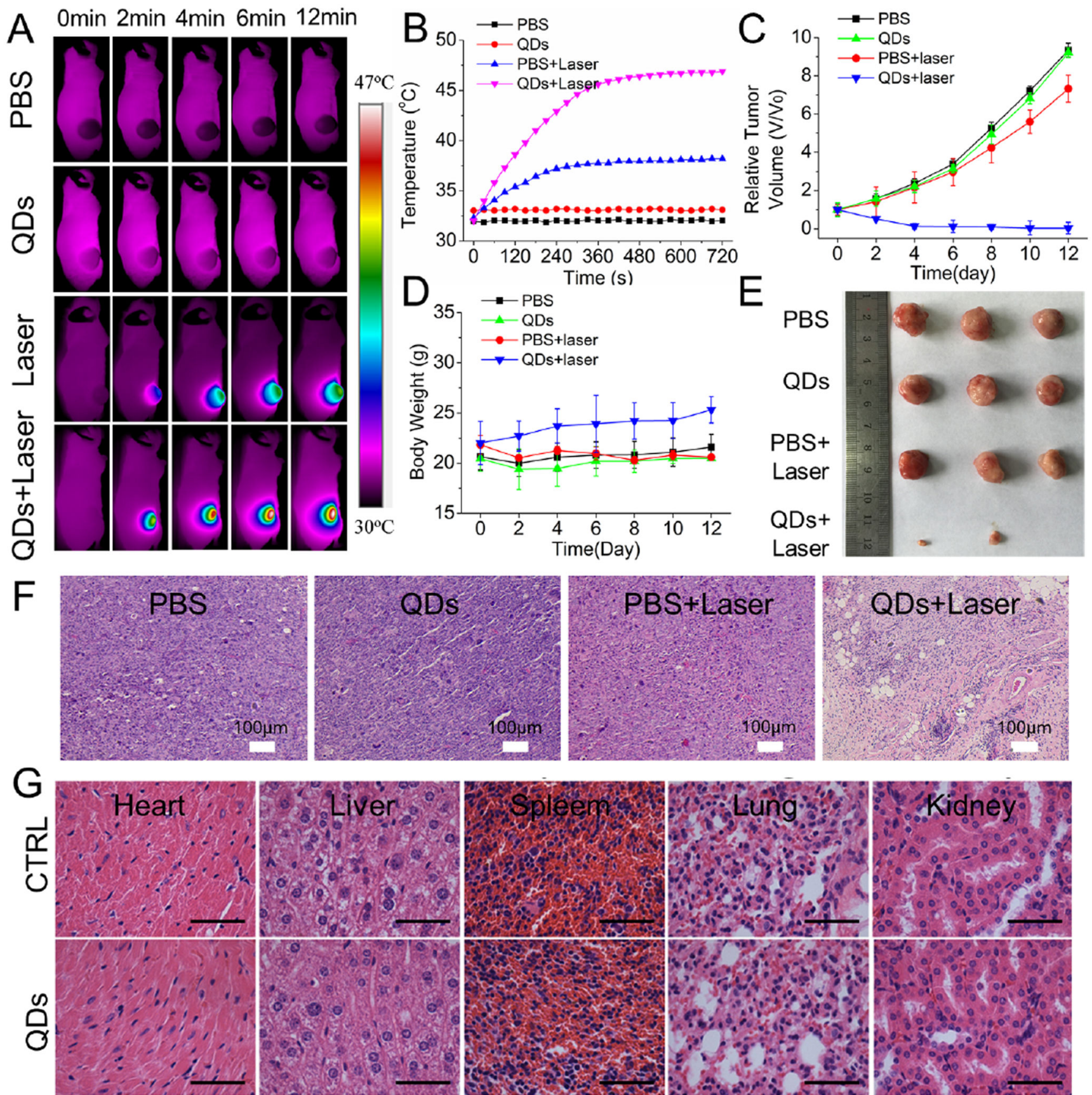

Figure 5.

In vivo thermal imaging and PTT. (A) Thermal IR imaging of 4T1 tumor-bearing mice after i.v. injection of ZCIS NMs-25 and exposure to $660 \mathrm{~nm}$ laser irradiation. (B) Temperature change curves of $4 \mathrm{~T} 1$ tumors in mice treated with or without ZCIS NMs-25 and laser irradiation as a function of irradiation time. (C) Growth curves of tumors in mice from the different treatment groups. Tumor volumes were normalized to their initial sizes. Error bars represent the standard deviations of 3 mice per group. (D) Body weight curves of mice in the different treatment groups. (E) Digital photos and (F) H\&E staining of tumor tissues collected from mice in the different groups at the end of treatment. (G) H\&E-stained slices 
of the heart, liver, spleen, lung, and kidney in mice without and with PTT treatment. Scale bar $=50 \mu \mathrm{m}$. 